\title{
A Transit-Based Evacuation Model for Metropolitan Areas
}

\author{
Xin Zhang, Gang-len Chang \\ University of Maryland
}

\begin{abstract}
This paper develops a decision-support model for transit-based evacuation planning occurring in metropolitan areas. The model consists of two modules executed in a sequential manner: the first deals with determining pick-up locations from candidate locations based on the spatial distribution of the evacuees, and the second plans for the route and schedule for each transit vehicle based on vehicle availability and evacuee demand pattern. An overlapping clustering algorithm is first adopted in allocating the demands to several nearby clusters. Then, an optimization model is proposed to allocate available buses from the depots to transport the assembled evacuees between the pick-up locations and different safety destinations and public shelters. A numerical example based on the city of Baltimore demonstrates the applicability of the proposed model and the advantages compared to state-of-the-art models with overly strict and unrealistic assumptions.
\end{abstract}

\section{Introduction}

Under potential terrorist attack, harmful substances released from transportation and industrial accidents, fires, floods, and other emergencies require immediate evacuation from hazardous areas. In congested metropolitan areas, commuters are likely to depend on either transit or other modes for their daily commute and, thus, may not have access to their private vehicles. Once an incident occurs, responsible agencies, such as city transportation administration and emergency units, should quickly devise a plan to dispatch available public transit resources to evacuate carless populations.

Although aiding the carless during evacuations has been presented in recent research, planning details such as how to identify available buses and drivers, how to determine potential pick-up locations, how to allocate bus routes to collect evacuees, and how to provide a timetable for the drivers (USDOT and USDHS 2006) are not well studied. Litman (2006) and Renne (2008) highlighted the needs for transit-dependent people during evacuation planning based on their experience with hurricanes Katrina and Rita. Wolshon (2001) mentioned that about 15-30 percent of the evacuation population in New Orleans during the hurricanes was transit-dependent. Fittante (2012) demonstrated community transit's value in response to Hurricane Sandy. Transit agencies should be in a position 
to render valuable assistance during emergencies. During the 9/11 terrorist attack, the transit system in New York City allowed free entry and led evacuees to safe locations. In Washington DC, buses contributed to the response effort, and additional buses were provided to the DC police to move officers to key locations. The Federal Transit Administration (FTA) and other agencies have issued many security guidelines for response before, during, and after a threat to ensure a quick recovery (FTA 2002; USDOT 2006). It is suggested that transit agencies to perform their own review on performance indicators for assessing emergency preparedness (Nakanishi 2003).

The transit evacuation model developed in this paper concerns the evacuation scenario occurring in metropolitan areas-for example, under a no-notice threat during a football game. The model consists of two modules executed in a sequential manner: the first deals with determining pick-up locations from candidate locations based on the spatial distribution of the evacuees. Once the pick-up locations are set, the evacuees are allocated accordingly, and the arrival pattern of each pick-up location is obtained. Fuzzy c-means (FCM), developed by Dunn (1974) and improved by Bezdek (1984), is applied to allow people at one location to be assigned to multiple clusters. The second module develops an integer-linear optimization module and plans the route and schedule for each transit vehicle based on vehicle availability and evacuee demand patterns.

\section{Literature Review}

Various studies have focused on different aspects of evacuation planning, such as demand modeling (Mei 2002; Wilmot 2004; Fu 2007), departure scheduling (Malone 2001; Mitchell 2006; Sbyati 2006; Chien 2007; Chen 2008), route choice (Cova 2003; Afshar 2008; Chiu 2008; Yazici 2010; Zheng 2010; Xie 2011; ), contra-flow operation (Theodoulou 2004; Wolshon 2005; Tuydes 2006; Xie 2010) and relief operation (Haghani 1996; Barbarosoglu 2004; Ozbay 2007; Xie 2009). Most of these are specific to the control and management of passenger car flows. Compared to these evacuation research efforts, there are only a limited number of studies on modeling transit-based evacuation. Elmitiny (2007) simulated different strategies and alternative plans for the deployment of transit during an emergency situation. Chen (2009) proposed a bi-level optimization model to determine waiting locations and corresponding shelters in a transit-based evacuation; the model was applied on the network within the University of Maryland. Song (2009) formulated transit evacuation operation during a natural disaster as a location-routing problem aiming to minimize total evacuation time; the problem identified the optimal serving area and transit vehicle routings to move evacuees to safety shelters. Abdelgawad (2010) developed an approach to optimally operate the available capacity of mass transit to evacuate transit-dependent people during no-notice evacuation of urban areas; an extended vehicle routing problem was proposed to determine the optimal scheduling and routing for the buses to minimize the total evacuation time. Sayyady (2010) proposed a mixed-integer linear program to model the problem of finding optimal transit routes during no-notice disasters; a Tabu-search algorithm was designed and an experiment was conducted using the transportation network of Fort Worth, Texas. Naghawi (2010) systematically modeled and simulated transit-based evacuation strategies applying the TRANSIMS agent-based transportation simulation system to the assisted evacuation plans of New Orleans. Kaisar 
(2012) addressed the optimal allocation of bus stops for the purpose of evacuating special needs populations; to evaluate the solution quality, a microscopic traffic simulation model was developed to represent the downtown Washington DC area in an evacuation scenario.

In carefully examining the similar study efforts, it can be concluded that most of the above models assumed one or several of the following:

- The pick-up locations serving as convening points are given and known in advance.

- All evacuees are present at the pick-up locations shortly after the evacuation starts.

- The loading and unloading times at pick-up locations are negligible or are assumed to be a constant value.

- Each vehicle is assigned a fixed route and runs in a cycle.

- The destinations have infinite holding capacities for evacuees.

Most of these assumptions are over-restrictive and, thus, prevent the application of the model outcome to real-world evacuation scenarios. To overcome these restrictions, our model tries to relax these assumptions and has the following unique characteristics that distinguish it from the previous studies:

- Both pick-up location allocation and transit bus scheduling are considered. During evacuations, the massive number of evacuees first needs to be coordinated and guided to nearby convene points, and then the transit vehicles are scheduled depending on the time-dependent arrival patterns of the evacuees at these pick-up points.

- The demand pattern is treated as time-dependent. Most prior research assumes that all evacuees are queued at pick-up locations at the beginning of the evacuation. This is almost never true because the evacuees may begin to evacuate at different times and it takes time for them to walk to the designated pick-up locations, and also because the pick-up locations, such as bus stops, have limited holding capacities, thus accumulating crowds and causing a huge bottleneck at that location.

- The loading/unloading times depend on the actual boarding/deboarding times. Negligence of this will overestimate the transport efficiency in generating the bus route and scheduling the timetables.

- Although pick-up locations are determined beforehand, the bus route is more flexible than the daily fixed route, servicing different pick-up locations at different runs based on actual need. Sometimes it is inefficient for a bus route to service fixed pick-up locations back and forth during evacuations. Instead, once a bus drops off evacuees at a safety area, it will be dispatched to the most-needed pick-up location.

- Capacity constraint is incorporated into destinations that are commonly public shelters, such as stadiums, schools, parks etc. Without such constraint, the model is subject to generate solutions in which all evacuees are sent to one or two nearest shelters and may cause overcrowding problems. 


\section{Modeling Pick-Up Location Selection}

The set of pick-up locations should cover all carless evacuees and limit their total walking distance, as per FTA requirements. This study grouped all evacuee generation points into several clusters of demand zones, and then allocated a pick-up location within each zone. For each demand point, the evacuees were distributed based on the proximity of the nearby pick-up location within walking range. An overlapping clustering algorithm to tie a particular demand point to several nearby clusters was adopted. Developed by Dunn (1974) and improved by Bezdek (1984), Fuzzy c-means (FCM) allows one piece of data to be assigned to multiple clusters, which is based on the following objective function:

$$
J=\sum_{i=1}^{N} \sum_{j=1}^{C} u_{i j}\left\|x_{i}-c_{j}\right\|^{2}
$$

where,

$$
\begin{aligned}
& x_{i}=\text { the ith measured data } \\
& c_{j}=\text { the center of the cluster } \\
& u_{i j}=\text { the degree of membership of } x_{i} \text { in the cluster } j \\
& \|*\|=\text { the second-norm expressing the similarity between measured data and the } \\
& \quad \text { center }
\end{aligned}
$$

In the context of determining bus pick-up locations, $x_{i}$ is the ith evacuee's position, $c_{j}$ is the jth pick-up location, $\|*\|$ is the distance between the evacuee and the pick-up location, and $u_{i j}$ measures the likelihood the evacuee $i$ will move to the pick-up location $j$.

The entire algorithm for determining the pick-up locations and the evacuee's allocation plan is composed of the following steps:

(1) Initialize $c_{j}$ and $u_{i j}=\frac{1}{\sum_{k=1}^{C}\left(\frac{\left\|x_{i}-c_{j}\right\|}{\left\|x_{i}-c_{k}\right\|}\right)}$.

(2) Calculate $c_{j}=\frac{\sum_{i=1}^{N} u_{i j} x_{i}}{\sum_{i=1}^{N} u_{i j}}$.

(3) Calculate $u_{i j}=\frac{1}{\sum_{k=1}^{C}\left(\frac{\left\|x_{i}-c_{j}\right\|}{\left\|x_{i}-c_{k}\right\|}\right)}$.

(4) If $\Delta U=\max \left(\Delta u_{i j}\right) \leq \varepsilon$, go to step 5; otherwise, go to step 2 .

(5) If $\left\|x_{i}-c_{j}\right\|>\xi$, set. 
Note if any of the final pick-up locations is geographically feasible (e.g. river, rail-road, and building), then it needs to be adjusted to the closest geographically location, which can serve the pick-up purpose.

\section{Transit Vehicle Routing and Scheduling}

\section{Define Pick-Up Request and Vehicle Route}

Each pick-up request is associated with the following parameters: pick-up location, number of evacuees (usually equal to load capacity), and time-window with an upper and lower bound. The bound for the time window can ensure people being picked up on time and prevent intolerably long waiting times. For each pick-up location ${ }^{i}$, we divide the time horizon into time segments with lengths $t_{i j}, j=1,2,3 \ldots$, and let $d_{i j}$ be the demand reaching pick-up location ${ }^{i}$ during time interval $t_{i j}$, then $t_{i j}=\arg \min \left(d_{i j}=C, t_{i j}=W\right)$, where $C$ is the bus capacity and $W$ is the maximum waiting time. For each time segment $t_{i j}, j=1,2,3 \ldots$, a pick-up request node is created with a time window $\left(a_{i j}=\sum_{0<k<j} t_{i k}, b_{i j}=a_{i j}+W\right)$. For example, given the capacity and the maximum waiting time to be 20 passengers and 2 minutes, Figure 1 and Table 1 show the pick-up requests I to VI created from the cumulative arrival curves at a particular pick-up location $i$.

FIGURE 1.

Pick-up requests generated

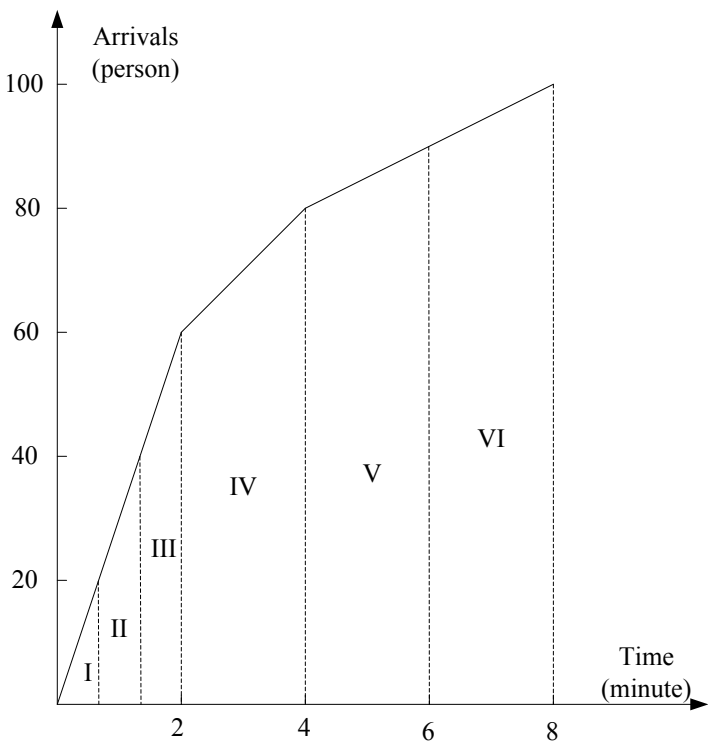

TABLE 1.

Details of Pick-Up Requests

\begin{tabular}{|c|c|c|}
\hline Pick-up Request & Time Window & Pick-up Number \\
\hline I & $0-120 \mathrm{~s}$ & 20 \\
\hline II & $40-160 \mathrm{~s}$ & 20 \\
\hline III & $80-200 \mathrm{~s}$ & 20 \\
\hline IV & $120 \mathrm{~s}-240 \mathrm{~s}$ & 20 \\
\hline V & $240-360 \mathrm{~s}$ & 10 \\
\hline VI & $360-480 \mathrm{~s}$ & 10 \\
\hline
\end{tabular}


Define $N^{P}$ be the set of pick-up request nodes, $N^{O}$ be the set of origin nodes for buses, $N^{D}$ be the set of destination nodes for drop off, and $N^{E}$ be the set of the end depot nodes where the bus mission is completed upon arrival. Each pick-up request node $i$ is associated with a time window $\left[a_{i}, b_{i}\right]$. Herein we consider the hard time window so that ${ }^{i}$ must be visited by a bus before $b_{i i}$. Let $V$ be a set of homogenous buses to be used in evacuation. The route for each bus is designed in the following manner: pull out of $N^{O}$; visit several (no more than two in this study) pickup-request nodes, satisfying their time windows, and go to a destination node to drop off; come back to visit another pick-up request node within the time window; go to another destination node to drop off, and so on, until no pickup-request can be satisfied within allowed time window; finally, go to the end-depot. Each bus is dispatched in such a manner until all pickup-requests are visited exactly once.

Figure $2 \mathrm{a}$ shows the typical route for one bus, which consists of multiple runs. Each run includes one or two pick-up request nodes and one drop off node. Figure $2 b$ illustrates two example bus routes. We tried to assign buses starting from $N^{O}$ and visiting nodes in $N^{P}$ and $N^{D}$ alternately and continuously.

FIGURE 2.

Illustration of bus routes

One trip

Start-depot $\longrightarrow$ Pick-up requests $\longrightarrow$ Drop-off $\longrightarrow$
Multiple trips

h

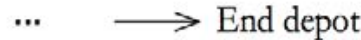

(a) Typical route for one bus

Set of start-depots Set of pickup-requests Set of drop-off points End depot

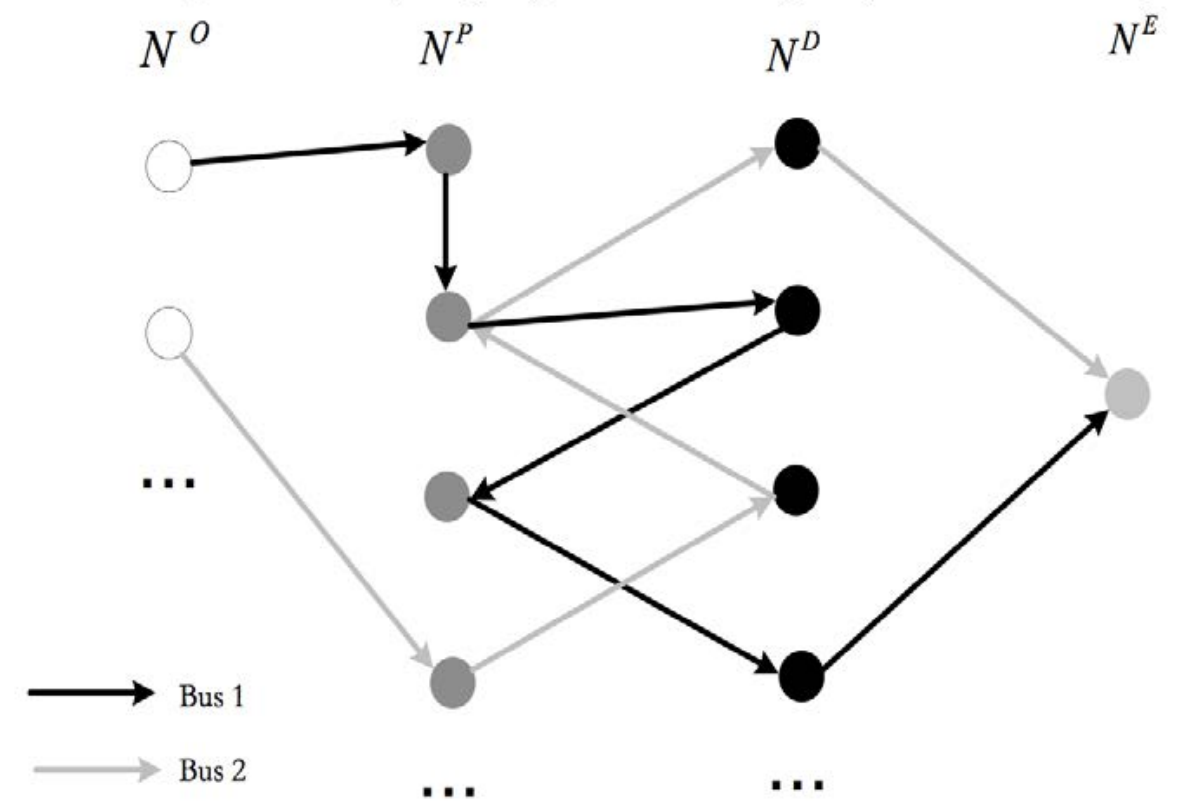

(b) Bus routes 


\section{Variable Definition}

The decision variables employed in this model are defined below. The indicator variables represent the sequence of the bus routes, and the other integer variables represent the arrival times, departure times, and the bus loads.

$b_{i, j, k}=$ indicator whether bus $\mathrm{k}$ moves from node $\mathrm{i}$ to $\mathrm{j}$

$b_{i, j, m, k}=$ indicator whether bus $\mathrm{k}$ moves from node $\mathrm{i}$ to destination $\mathrm{j}$ and then to node $\mathrm{m}$

$w_{i, k, i}=$ indicator whether node $\mathrm{i}$ is routed by the kth vehicle at lth run

$t_{i, k}^{A}=$ the time bus $\mathrm{k}$ arrives at request node $\mathrm{i}$

$t_{i, k}^{D}=$ the time bus $\mathrm{k}$ departs from request node $\mathrm{i}$

$t_{i, j, m, k}^{A}=$ the time bus $\mathrm{k}$ arrives at destination node $\mathrm{j}$ following node $\mathrm{i}$ and preceding node $\mathrm{m}$

$t_{i, j, m, k}^{D}=$ the time bus $\mathrm{k}$ departs from destination node $\mathrm{j}$ following node $\mathrm{i}$ and preceding node $\mathrm{m}$

$l_{k, l}=$ the load for bus $\mathrm{k}$ at run 1

$l_{i, k, l}=$ the load to destination $\mathrm{i}$ for bus $\mathrm{k}$ at run 1

The known variables are defined as follows:

$N^{O}=$ the set of origin nodes for buses, e.g. bus depots

$N^{D}=$ the set of destination nodes for evacuees, e.g. shelters

$N^{P}=$ the set of pick-up request nodes for evacuees, e.g. shelters

$N^{E}=$ the set of virtual end depots

$T T_{i, j}=$ the travel time from node $\mathrm{i}$ to node $\mathrm{j}$

$n_{i}=$ the number of pedestrians for request $\mathrm{i}$

$L_{\max }=$ the maximum load of each bus

$C_{i}=$ the capacity of destination $\mathrm{i}$

$a_{i}=$ the lower bound of the time window of node $\mathrm{i}$

$b_{i}=$ the upper bound of the time window of node $\mathrm{i}$

\section{Mathematical Formulation}

\section{Objective Function}

The objective of the model in equation (2) is to minimize the time for the last evacuees to arrive at safe destinations. The definition of the time window for the pick-up request guaranteed that the evacuees would not wait more than the maximum waiting time to board the bus.

$$
\text { Minimize } \max \left(t_{m, k}^{A}\right), \forall m \in N^{E}, \forall k \in K
$$


The model formulation also includes travel time constraints, time window constraints, pick-up requests constraints, bus load constraints, and destination capacity constraints, which are detailed in the following sections.

\section{Travel Time Constraints}

$$
\begin{aligned}
& t_{j, k}^{A}-t_{i, k}^{D} \leq T T_{i, j}+M\left(1-b_{i, j, k}\right), \forall i \in N^{P} \cup N^{O}, j \in N^{P}, \forall k \in K \\
& t_{j, k}^{A}-t_{i, k}^{D} \geq T T_{i, j}-M\left(1-b_{i, j, k}\right), \forall i \in N^{P} \cup N^{O}, \forall j \in N^{P}, \forall k \in K \\
& t_{i, j, m, k}^{A}-t_{i, k}^{D} \leq T T_{i, j}+M\left(1-b_{i, j, m, k}\right), \forall i \in N^{P}, \forall j \in N^{D}, \forall k \in K \\
& t_{i, j, m, k}^{A}-t_{i, k}^{D} \geq T T_{i, j}-M\left(1-b_{i, j, m, k}\right), \forall i \in N^{P}, \forall j \in N^{D}, \forall k \in K \\
& t_{m, k}^{A}-t_{i, j, m, k}^{D} \leq T T_{j, m}+M\left(1-b_{i, j, m, k}\right), \forall i \in N^{P}, \forall j \in N^{D}, \forall m \in N^{P} \cup N^{E}, \forall k \in K \\
& t_{m, k}^{A}-t_{i, j, m, k}^{D} \geq T T_{j, m}-M\left(1-b_{i, j, m, k}\right), \forall i \in N^{P}, \forall j \in N^{D}, \forall m \in N^{P} \cup N^{E}, \forall k \in K
\end{aligned}
$$

Constraints (3) and (4) set the travel time needed from the pick-up node i to j, constraints (5) and (6) set the travel time from the pick-up node i to destination node j, and constraints (7) and (8) set the travel time from destination node $\mathrm{j}$ to pick-up node $\mathrm{m}$.

\section{Time Window Constraint}

$$
\begin{aligned}
& t_{i, k}^{D}-t_{i, k}^{A} \geq n_{i} \Delta t, \forall i \in N^{p}, \forall k \in K \\
& t_{i, j, m, k}^{D}-t_{i, j, m, k}^{A} \geq l_{k, l} \Delta t-M w_{j, k, l}, \forall i, j, m \in N, \forall k \in K, \forall l \in L \\
& t_{i, k}^{A} \leq b_{i}, \forall i \in N^{p} \\
& t_{i, k}^{D} \geq a_{i}, \forall i \in N^{p}
\end{aligned}
$$

Constraint (9) considers the loading time at the pick-up request node i. Constraint (10) calculates the unloading time needed at the destination node j based on the bus load. Constraints (11) and (12) force the arrival time to be earlier than the upper bound of the time window and the departure time to be later than the lower bound of the time window at the pick-up request $\mathrm{i}$.

Each pickup request can be served by only one bus:

$$
\begin{aligned}
& \sum_{i} \sum_{k} b_{i, j, k}=1, \forall j \in N^{p} \\
& \sum_{j} \sum_{k} b_{i, j, k}=1, \forall i \in N^{p} \\
& \sum_{j} b_{i, j, k}=1, \forall i \in N^{O}, \forall k \in K
\end{aligned}
$$




$$
\begin{aligned}
& \sum_{m \in N^{P}} b_{i, j, m, k}=1, \forall i \in N^{P}, \forall j \in N^{D}, \forall k \in K \\
& \sum_{i \in N^{P}} b_{i, j, m, k}=1, \forall m \in N^{P}, \forall j \in N^{D}, \forall k \in K \\
& b_{i, j, m, k}=0, \forall j \in N^{E} \\
& \sum_{k} \sum_{l} w_{i, k, l}=1, \forall i \in N^{p} \\
& b_{i, j, m, k} \leq b_{i, j, k}+b_{j, m, k}-0.95 \\
& b_{i, j, m, k} \geq b_{i, j, k}+b_{j, m, k}-1.01 \\
& w_{i, k, l}-w_{j, k, l} \leq M\left(1-b_{i, j, k}\right) \\
& w_{i, k, l}-w_{j, k, l} \geq M\left(b_{i, j, k}-1\right)
\end{aligned}
$$

Constraints (13) and (14) ensure each pick-up request is serviced exactly once. Constraint (15) ensures that the bus from the depot can head to only one pick-up location. Constraints (16) and (17) ensure only one preceding and following node for the destination node at each bus run. Constraint (18) dismisses the bus mission once it reaches the end depot. Constraint (19) ensures that each pick-up request be serviced exactly once. Constraints (20) and (21) ensure that the value of $b_{i, j, m, k}$ can be 1 only if $b_{i, j, k}$ and $b_{j, m, k}$ are both 1 . Constraints (22) and (23) establish the relationship between the indicator variables $b$ and $w$, which means that if pick-up request $j$ is serviced by one bus followed by pick-up request $i$, then the indicator variable $w$ value should be identical for pick-up requests $i$ and $\mathrm{j}$ for the same bus run.

\section{Bus Capacity Constraints}

$$
\begin{aligned}
& l_{k, l}=\sum_{i} n_{i} w_{i, k, l}, \forall k \in K, \forall l \in L \\
& l_{k, l} \leq L_{\max }, \forall k \in K, \forall l \in L
\end{aligned}
$$

Constraint (24) calculates the total load for the bus $k$ at the lth run, and constraint (25) limits the load to be less than the maximum load for each bus.

\section{Destination Capacity Constraints}

$$
\begin{aligned}
& l_{i, k, l} \geq l_{k, l}-M\left(1-w_{i, k, l}\right), \forall i \in N^{D}, \forall k \in K, \forall l \in L \\
& l_{i, k, l} \leq l_{k, l}+M\left(1-w_{i, k, l}\right), \forall i \in N^{D}, \forall k \in K, \forall l \in L \\
& l_{i, k, l} \leq M w_{i, k, l}, \forall i \in N^{D}, \forall k \in K, \forall l \in L \\
& l_{i, k, l} \geq-M w_{i, k, l}, \forall i \in N^{D}, \forall k \in K, \forall l \in L \\
& \sum_{k} \sum_{l} l_{i, k, l} \leq C_{i}, \forall i \in N^{D}
\end{aligned}
$$


Constraints (26)-(29) calculate the number of evacuees unloaded at the destination i at the $1^{\text {st }}$ run for bus $k$. Constraint (30) limits the total unloaded evacuees at each destination node to be less than its holding capacity.

\section{Further Simplification}

Note that, during evacuations, the evacuee volumes are high, especially at beginning. The number of evacuees at a single pick-up request is very likely to be close to or reach bus capacity. In addition, some pick-up requests may have restrictive time windows, such that the bus serving one of these types of request nodes will not have enough remaining capacity or time to service any other pick-up request at that run. Thus, we can divide the pick-up request nodes into two groups: $N_{1}^{p}$ and $N_{2}^{p}=N^{p} \backslash N_{1}^{p}$. Any node $\mathrm{i}$ in the group $N_{1}^{p}$ and any node j in $N^{p}$ satisfies at least one of the following criteria:

(1) $n_{i}+n_{j}>L_{\max }$

(2) $\left[a_{i}+n_{i} \Delta t+T T_{i j}, b_{i}+n_{i} \Delta t+T T_{i j}\right] \cap\left[a_{j}, b_{j}\right]=\varnothing$ and

$$
\left[a_{j}+n_{j} \Delta t+T T_{j i}, b_{j}+n_{j} \Delta t+T T_{j i}\right] \cap\left[a_{i}, b_{i}\right]=\varnothing
$$

The pick-up request in $N_{1}^{p}$ either has a close-to-capacity number of pick-ups or an inflexible time window, which cannot accommodate other requests and thus should be serviced exclusively by one run. Constraint (31) excludes the possibility of servicing any two pick-up requests within $N_{1}^{p}$, which simplifies the formulation and, in turn, improves the computation speed.

$$
b_{i, j, k}=0, \forall i, j \in N_{1}^{P}, \forall k \in K
$$

\section{Numerical Example}

The model was tested on the city of Baltimore's downtown road network. A hypothetical evacuation after a sudden incident such as a terrorist attack was assumed. Figure 3 shows the spatial distribution of the demand points, bus depots, and safety destinations based on the aggregated 2010 MPO data from Baltimore County. There were around 40 pedestrian demand points, 2 transit depots, and 10 safety shelters in the vicinity area of the downtown area. The sizes of the demand points indicate the levels of the evacuee numbers at the locations and were estimated based on the traffic analysis data provided by Baltimore County. The two bus depots were the Bush Bus Division in the southwest and the Kirk Bus Division in the northeast and included high schools, community colleges, recreation centers, etc. For illustrative purposes, a constant evacuation rate every 10 minutes for the first 30 minutes was assumed at any given demand location. CPLEX 12.4 was adopted to solve the mixed-integer programming problem on a Windows7 computer with an Intel i-7 3770 CPU and 8GB of memory. 
FIGURE 3.

Demand points, depots, and safety locations

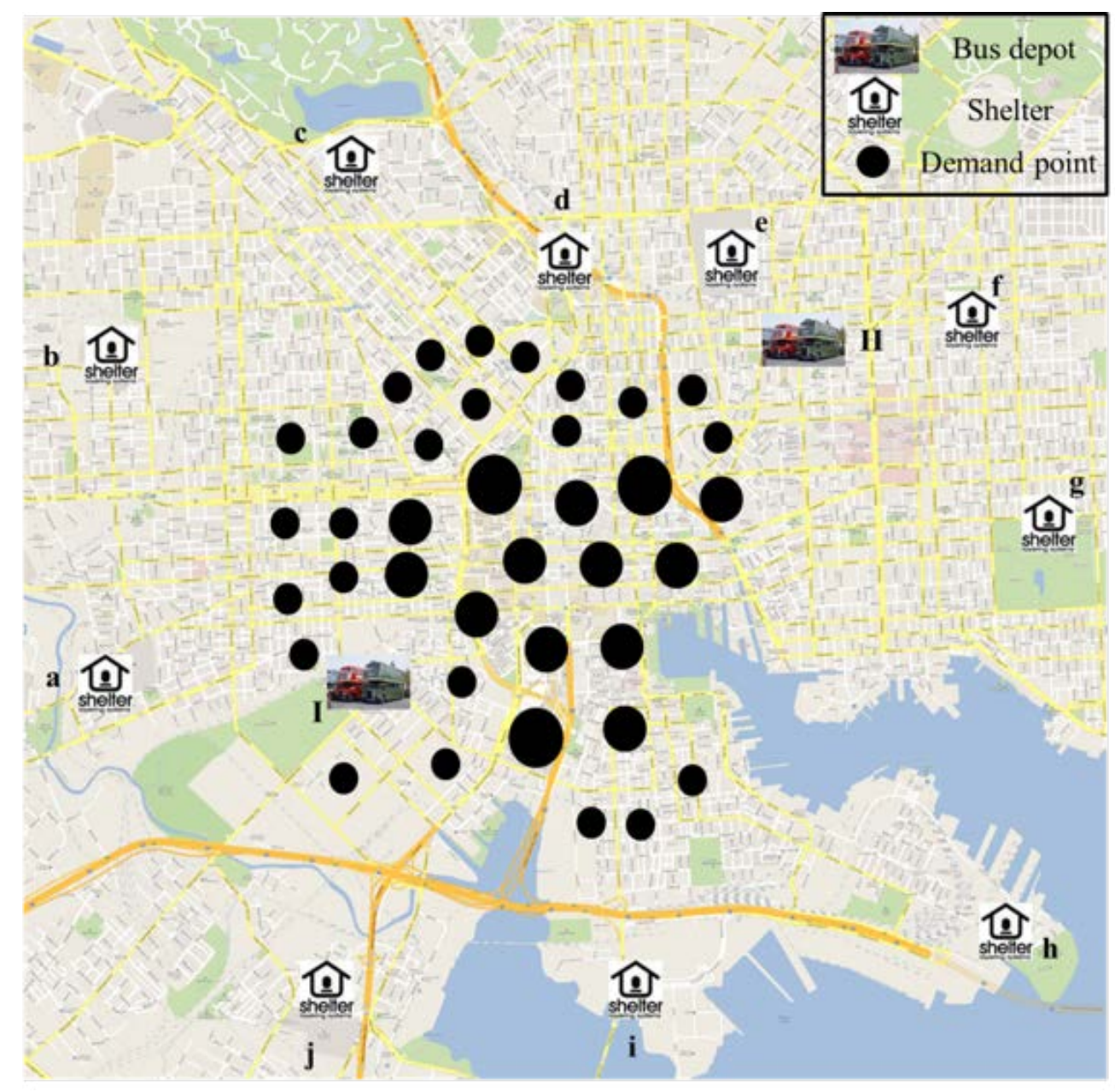

Table 2 lists the rate ranges in terms of pedestrian level, the capacities of the shelters, and the availability of buses in the depots. Figure 4 depicts the 11 candidate pick-up locations after the fuzzy clustering on the evacuee demand points. The average walking time for the evacuees to the pick-up locations was 3.1 minutes; the farthest was 9.8 minutes. Table 3 shows the cumulative arrival curve for the 11 pick-up locations based on the pedestrian levels and the degrees of membership of their nearby demand locations. Figure 5 shows two among the generated bus routes, and Table 4 lists the corresponding time and pick-up schedules. 
TABLE 2.

Shelter Capacities,

Bus Availabilities, and Demand Levels

\begin{tabular}{|c|c|c|c|}
\hline Shelter ID & Capacity (person) & Depot ID & Bus Availability \\
\hline $\mathrm{a}$ & 1,500 & $\mathrm{I}$ & 50 \\
\hline $\mathrm{b}$ & 1,000 & $\mathrm{I}$ & 50 \\
\hline $\mathrm{c}$ & 1,500 & & \\
\hline $\mathrm{d}$ & 2,000 & & \\
\hline $\mathrm{e}$ & 1,000 & & \\
\hline $\mathrm{f}$ & 1,000 & & \\
\hline $\mathrm{g}$ & 1,000 & Pedestrian level & Rate for first 10 min (person/min) \\
\hline $\mathrm{h}$ & 1,000 & High & 30 \\
\hline $\mathrm{i}$ & 1,000 & Medium & 20 \\
\hline $\mathrm{j}$ & 1,000 & Low & 10 \\
\hline
\end{tabular}

FIGURE 4.

Pick-up locations after fuzzy clustering

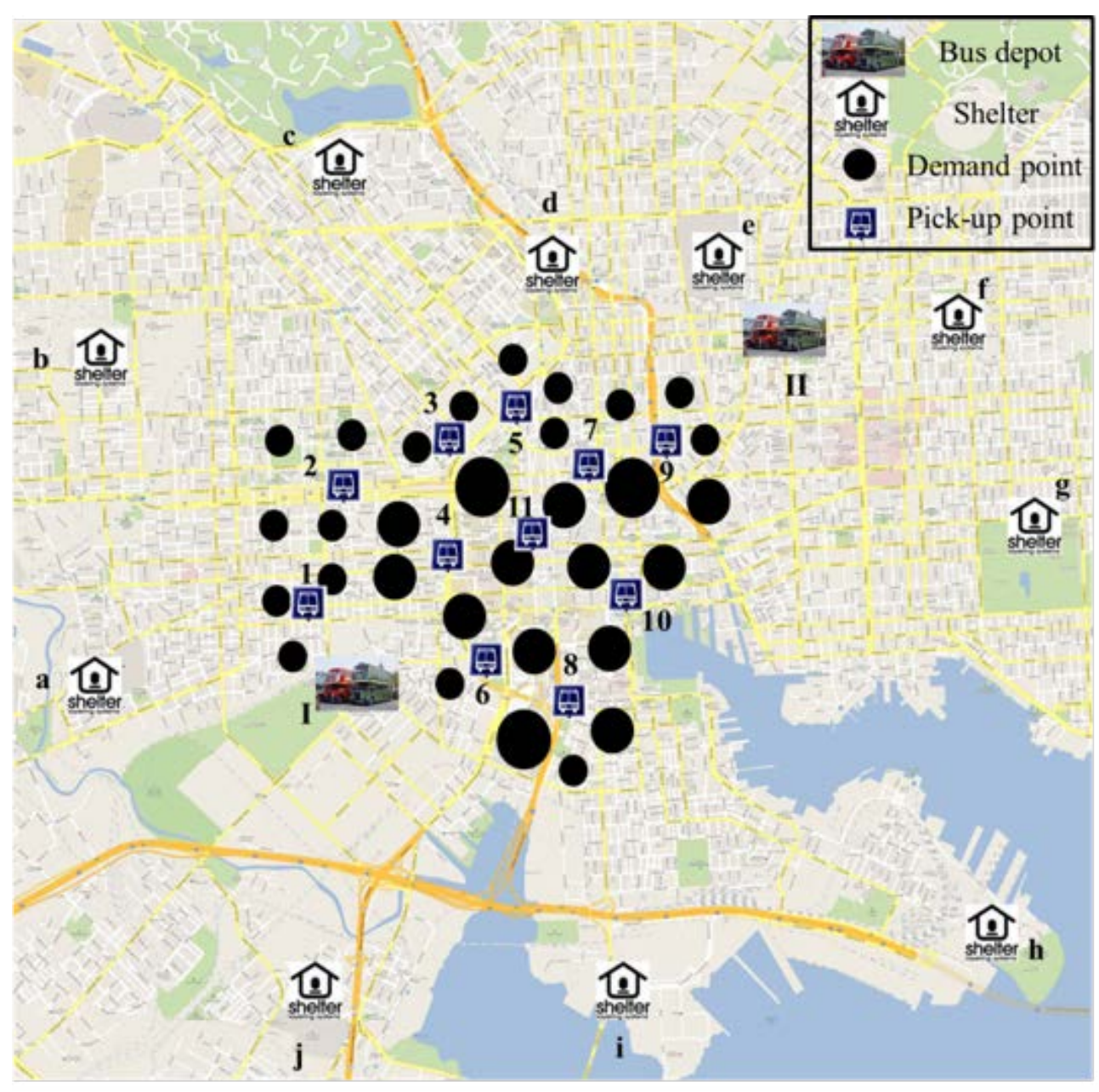


TABLE 3.

Time-Dependent Arrivals to Pick-Up Locations

\begin{tabular}{|c|c|c|c|c|}
\hline Pick-up Location & $\mathbf{0 - 1 0}(\mathbf{m i n})$ & $\mathbf{1 0 - 2 0}(\mathbf{m i n})$ & $\mathbf{2 0 - 3 0}(\mathbf{m i n})$ & $\mathbf{3 0 - 4 0}(\mathbf{m i n})$ \\
\hline 1 & 269 & 211 & 105 & 23 \\
\hline 2 & 353 & 271 & 136 & 30 \\
\hline 3 & 353 & 272 & 135 & 31 \\
\hline 4 & 362 & 265 & 130 & 23 \\
\hline 5 & 265 & 200 & 106 & 20 \\
\hline 6 & 363 & 268 & 133 & 26 \\
\hline 7 & 450 & 355 & 180 & 34 \\
\hline 8 & 628 & 478 & 256 & 45 \\
\hline 9 & 639 & 415 & 208 & 35 \\
\hline 10 & 365 & 261 & 135 & 20 \\
\hline 11 & 451 & 352 & 176 & 34 \\
\hline
\end{tabular}

FIGURE 5.

Two examples of generated bus routes.

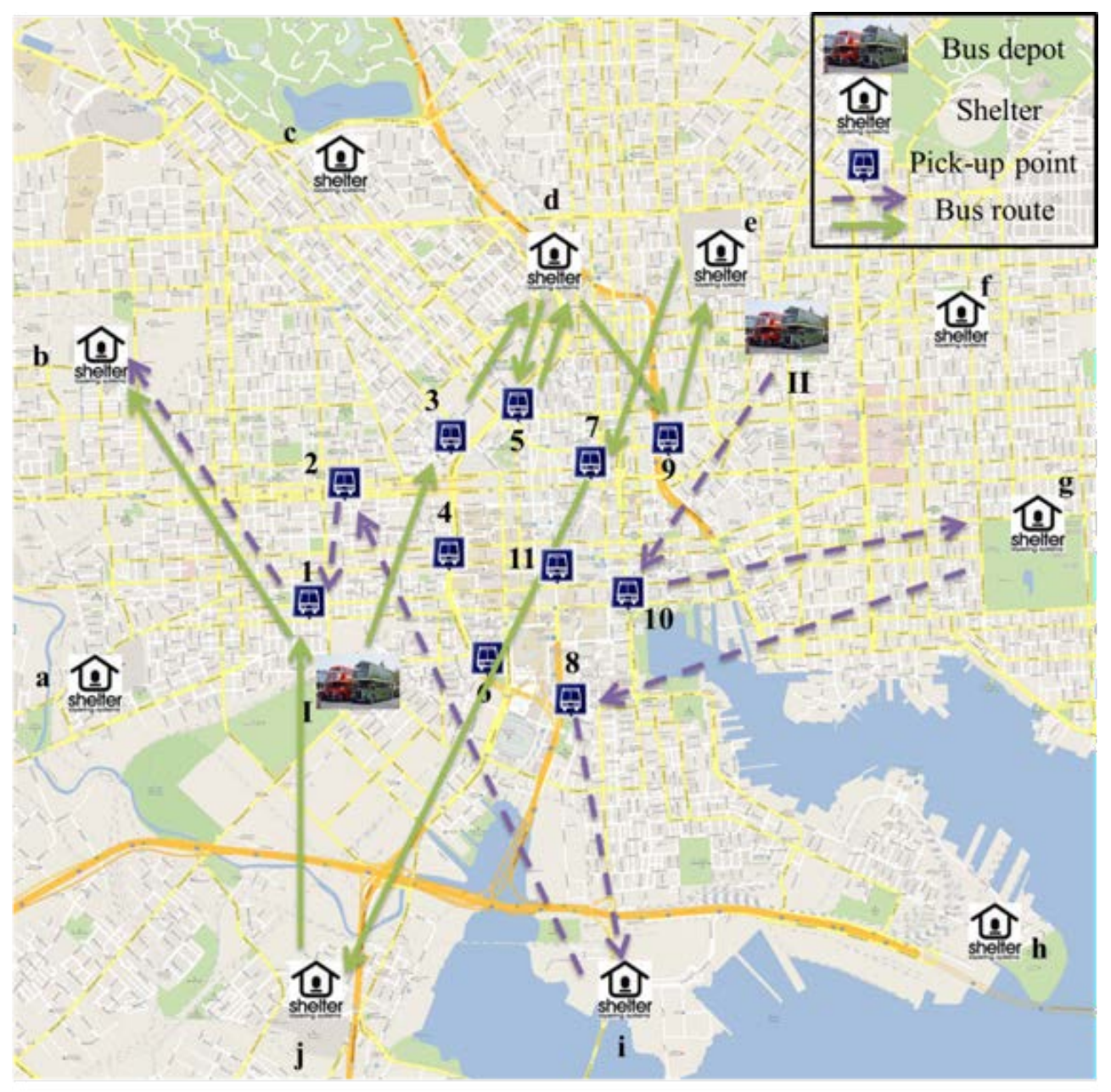


TABLE 4.

Time and Load Schedules

for Bus Routes

\begin{tabular}{|c|c|c|c|c|c|c|}
\hline Bus & Run & Depot & $\begin{array}{c}\text { Pick-up } \\
\text { Node }\end{array}$ & Destination & $\begin{array}{l}\text { Bus load } \\
\text { (persons) }\end{array}$ & $\begin{array}{l}\text { Travel Time } \\
(\min )\end{array}$ \\
\hline \multirow{3}{*}{1} & 1 & \multirow{3}{*}{ II } & 10 & g & 50 & 12 \\
\hline & 2 & & 8 & i & 50 & 14 \\
\hline & 3 & & 2,1 & $b$ & 40 & 18 \\
\hline \multirow{5}{*}{2} & 1 & \multirow{5}{*}{1} & 3 & $d$ & 50 & 10 \\
\hline & 2 & & 5 & $d$ & 50 & 5 \\
\hline & 3 & & 9 & e & 50 & 7 \\
\hline & 4 & & 7 & j & 50 & 14 \\
\hline & 5 & & 1 & $b$ & 30 & 12 \\
\hline
\end{tabular}

As indicated previously, the improvements of this model compared to the previous studies are as follows:

- The time window of the pick-up request was used to restrict the maximum waiting time for evacuees.

- Pick-up nodes and targeted destinations do not have to be fixed in different runs.

- A time-dependent arrival curve at the pick-up locations is considered rather than assuming all evacuees are present at the location at the start.

To show the advantage of adopting the above improvements, we designed the following experiments:

- Use maximum waiting times of 2, 5, and 10 minutes.

- Fix the route in each bus run.

- Assume a full-demand start at pick-up locations.

Table 5 shows the minimum number of buses needed for the combination of the experiment settings. It can be seen that the longer the waiting time toleration, the fewer the number of buses are needed to service all evacuees. The strategy of fixing the route requires more vehicle resources than that of the flexible route. Assuming a full demand start, the number of buses needed is much higher, and most of the buses are scheduled simultaneously at the start of the evacuation, which may create a great burden on vehicle road traffic. The fixed and flexible route strategies under the full demand scenario do not make much difference, simply because most of the vehicles will be scheduled only for one run to meet the time window constraints.

TABLE 5.

Minimum Buses Required for Different Combination

\begin{tabular}{|c|c|c|c|}
\hline \multirow{2}{*}{ Max Waiting } & \multicolumn{2}{|c|}{ Arrival Curve } & Full Demand \\
\cline { 2 - 4 } & Fixed Route & Flex Route & Fixed Route \\
\hline 2 min & 87 & 73 & 87 \\
\hline 5 min & 73 & 53 & 73 \\
\hline $10 \mathrm{~min}$ & 46 & 31 & 46 \\
\hline
\end{tabular}


Table 6 shows the distribution of evacuees to safety areas with and without destination capacity constraints. It can be seen clearly in the latter case that a few shelters closest to the pick-up locations become overcrowded and exceed their actual capacity. With the capacity constraints in the model, the evacuees are distributed more evenly among the destinations.

TABLE 6.

Minimum Buses Required for Different Combination of Settings

\begin{tabular}{|c|r|r|}
\hline \multirow{2}{*}{ Destination ID } & \multicolumn{2}{|c|}{ Capacity Constraints } \\
\cline { 2 - 3 } & With & Without \\
\hline $\mathrm{a}$ & 1,388 & 1,388 \\
\hline $\mathrm{b}$ & 790 & 790 \\
\hline $\mathrm{c}$ & 1,414 & 0 \\
\hline $\mathrm{d}$ & 2,000 & 3,414 \\
\hline $\mathrm{e}$ & 1,000 & 1,297 \\
\hline $\mathrm{f}$ & 297 & 0 \\
\hline $\mathrm{g}$ & 388 & 0 \\
\hline $\mathrm{h}$ & 800 & 0 \\
\hline $\mathrm{i}$ & 1,000 & 2,188 \\
\hline $\mathrm{j}$ & 790 & 790 \\
\hline
\end{tabular}

\section{Conclusions}

This paper proposed an optimization approach to determine pick-up locations for evacuees and allocate trips for buses for rescue purposes in transit-based evacuation planning. The proposed model was formulated as an integer linear program. In the model, evacuee demand points were clustered, and the center was defined as the pick-up location. Evacuees at each demand point were guided to nearby pick-up locations according to their proximity. The buses started from the bus depot to pick up evacuees and dropped them off at the safety area; after unloading, they headed towards other pick-up locations until all evacuees were picked up. An example using the Baltimore downtown area showed that the proposed model was more realistic and yielded better results compared to previous models under some given assumptions.

This research should be useful to planners, transit agencies, and emergency management officials, as effective and reliable transit evacuation planning is imperative and critical based on experiences from the past. For emergency management agencies, how to efficiently use available public transit resources without keeping citizens waiting too long is critical. The paper offers an analytical approach to provide answers to some of the issues for transit-based evacuation, such as the following: How many transit vehicles are needed and should be reserved in case of an emergency situation? How can a flexible rather than a fixed route for drivers be scheduled to increase evacuation efficiency? How can reasonable dispatch schedules for transit vehicles be generated to prevent unnecessary road congestion by sending all vehicles at once? How can pick-up points for emergency purposes be reasonably selected? In addition, since this model adopts a generalized approach and is based on a few location-specific assumptions, it can be applied to other cities as long as the input demand, road network, and transit data are present. 
Although much has been done in this paper regarding transit evacuation modeling, the study is still exploratory and can be further improved. The limitations of this model include the following:

- The separation of the two modules (pick-up location selection and transit route optimization) may render non-optimality of the entire system. However, the current difficulty of combining these two lies in over-complexity of the model.

- Without distinguishing the categories and groups of evacuees, it is hard to preclude the possibility that people without special needs may occupy spaces reserved for special-needs groups, such as persons with disabilities and children.

- Although the computation speed is acceptable on a citywide transit network for evacuation within a reasonable time window, the NP-hardness of the integer-linear formulation may have an impact on the computation efficiency in the application of statewide networks and time windows in days and weeks.

- The model inputs currently rely on planning MPO data. However, there are numerous daily visitors in study areas that may not be captured by the data. Moreover, the actual number of evacuees at the time of evacuation is somewhat unpredictable. All these factors may impact the optimal solutions.

- Current travel estimation is not based on real-time traffic information during evacuation. Few previous studies have tried to combine passenger car and transit evacuation modeling under a unified framework. Thus, the capability of estimating travel time during evacuations will affect the solution quality of this model.

To address these modeling limitations, future studies could focus on directions such as integrating the two decision modules, paying attention to special group needs, designing an efficient algorithm to expedite the computation process, performing sensitivity analyses, and receiving real-time input data feeds and integrating them with the passenger car evacuation model.

\section{References}

Abdelgawad, Hossam, and Baher Abdulhai. 2010. Managing large-scale multimodal emergency evacuations. Journal of Transportation Safety \& Security 2(2): 122-151.

Afshar, Abbas Mohasel, and Ali Haghani. 2008. Heuristic framework for optimizing hurricane evacuation operations. Transportation Research Record 2089(1): 9-17.

Barbarosog lu, G., and Y Arda, G. 2004. A two-stage stochastic programming framework for transportation planning in disaster response. Journal of the Operational Research Society 55(1): 43-53.

Bezdek, James C., Robert Ehrlich, and William Full. 1984. FCM: The fuzzy- c means clustering algorithm. Computers \& Geosciences 10(2): 191-203.

Chen, Xuwei, and Franklin B. Zhan. 2006. Agent-based modelling and simulation of urban evacuation: relative effectiveness of simultaneous and staged evacuation strategies. Journal of the Operational Research Society 59(1): 25-33. 
Chen, Cheng-Chieh Frank, and Chih-Sheng Chou. 2009. Modeling and performance assessment of a transit-based evacuation plan within a contraflow simulation environment. Transportation Research Record 2091(1), : 40-50.

Chien, Steven I., and Vivek V. Korikanthimath. 2007. Analysis and modeling of simultaneous and staged emergency evacuations. Journal of Transportation Engineering 133(3): 190-197.

Chiu, Yi-Chang, and Pitu B. Mirchandani. 2008. Online behavior-robust feedback information routing strategy for mass evacuation. Intelligent Transportation Systems, IEEE Transactions 9(2): 264-274.

Cova, Thomas J., and Justin P. Johnson. 2003. A network flow model for lane-based evacuation routing. 7 37(7): 579-604. Transportation Research Part A: Policy and Practice 37(7): 579-604.

Dunn, Joseph C. 1974. Well-separated clusters and optimal fuzzy partitions. Journal of Cybernetics 4(1): 95-104.

Federal Transit Administration. 2002. Transit threat level response recommendation, http://transit-safety.volpe.dot.gov/security/Securitylnitiatives/ThreatLevel/default. asp.

Fittante, Steve. 2012. Community transit demonstrates its value in response to Hurricane Sandy. Community Transportation.

Fu, Haoqiang, Chester G. Wilmot, Hong Zhang, and Earl J. Baker. 2007. Modeling the hurricane evacuation response curve. Transportation Research Record 2022(1): 94-102.

Haghani, Ali, and Sei-Chang Oh. 1996. Formulation and solution of a multi-commodity, multi-modal network flow model for disaster relief operations. Transportation Research Part A: Policy and Practice 30(3): 231-250.

Kaisar, Evangelos I., Linda Hess, and Alicia Benazir Portal Palomo. 2012. An emergency evacuation planning model for special needs populations using public transit systems. Journal of Public Transportation 15(2).

Litman, Todd. 2006. Lessons from Katrina and Rita: What major disasters can teach transportation planners. Journal of Transportation Engineering 132(1): 11-18.

Malone, Samuel W., Carl A. Miller, and Daniel B. Neill. 2001. Traffic flow models and the evacuation problem. UMAP Journal 22(3): 1-47.

Mei, B. 2002. Development of trip generation models of hurricane evacuation. Master's thesis, Louisiana State University, Baton Rouge.

Mitchell, Steven W., and Essam Radwan. 2006. Heuristic priority ranking of emergency evacuation staging to reduce clearance time. Transportation Research Record 1964(1): 219-228.

Naghawi, Hana, and Brian Wolshon. 2010. Transit-based emergency evacuation simulation modeling. Journal of Transportation Safety \& Security 2(2): 184-201. 
Nakanishi, Yuko, Kibeum Kim, Yavuz Ulusoy, and Andrew Bata. 2003. Assessing emergency preparedness of transit agencies: Focus on performance indicators. Transportation Research Record 1822(1): 24-32.

Ozbay, Kaan, and Eren Erman Ozguven. 2007. Stochastic humanitarian inventory control model for disaster planning. Transportation Research Record 2022(1): 63-75.

Renne, John L., Thomas W. Sanchez, and Todd Litman. 2008. National study on carless and special needs evacuation planning: A literature review.

Sayyady, Fatemeh, and Sandra D. Eksioglu. 2010. Optimizing the use of public transit system during no-notice evacuation of urban areas. Computers \& Industrial Engineering 59(4): 488-495.

Sbayti, Hayssam, and Hani S. Mahmassani. 2006. Optimal scheduling of evacuation operations. Transportation Research Record 1964(1): 238-246.

Song, Rui, Shiwei He, and Li Zhang. 2009. Optimum transit operations during the emergency evacuations. Journal of Transportation Systems Engineering and Information Technology 9(6): 154-160.

Theodoulou, Gregoris, and Brian Wolshon. 2004. Alternative methods to increase the effectiveness of freeway contraflow evacuation. Transportation Research Record 1865(1): 48-56.

Tuydes, Hediye, and Athanasios Ziliaskopoulos. 2006. Tabu-based heuristic approach for optimization of network evacuation contraflow. Transportation Research Record 1964(1): 157-168.

U.S. Department of Transportation, Research and Special Programs Administration. 2006. Public transportation system security and emergency preparedness planning guide.

U.S. Department of Transportation and U.S. Department of Homeland Security. 2006. Congress on catastrophic hurricane evacuation plan evaluation: A report to Congress.

Wilmot, Chester G., and Bing Mei. 2004. Comparison of alternative trip generation models for hurricane evacuation. Natural Hazards Review 5(4): 170-178.

Wolshon, Brian. 2001. One-way-out: Contraflow freeway operation for hurricane evacuation. Natural Hazards Review 2(3): 105-112.

Wolshon, Brian, Elba Urbina, Chester Wilmot, and Marc Levitan. 2005. Review of policies and practices for hurricane evacuation. I: Transportation planning, preparedness, and response. Natural Hazards Review 6(3): 129-142.

Wolshon, Brian, Elba Urbina Hamilton, Marc Levitan, and Chester Wilmot. Review of policies and practices for hurricane evacuation. II: Traffic operations, management, and control. Natural Hazards Review 6(3): 143-161.

Xie, Chi, and Mark A. Turnquist. 2009. Integrated evacuation network optimization and emergency vehicle assignment. Transportation Research Record 2091(1): 79-90. 
Xie, Chi, Dung-Ying Lin, and S. Travis Waller. 2010. A dynamic evacuation network optimization problem with lane reversal and crossing elimination strategies. Transportation Research Part E: Logistics and Transportation Review 46(3): 295-316.

Xie, Chi, and Mark A. Turnquist. 2011. Lane-based evacuation network optimization: An integrated Lagrangian relaxation and tabu search approach. Transportation Research Part C: Emerging Technologies 19(1): 40-63.

Yazici, Anil, and Kaan Ozbay. 2010. Evacuation network modeling via dynamic traffic assignment with probabilistic demand and capacity constraints. Transportation Research Record 2196, (1): 11-20.

Zheng, Hong, Yi-Chang Chiu, Pitu B. Mirchandani, and Mark Hickman. 2010. Modeling of evacuation and background traffic for optimal zone-based vehicle evacuation strategy. Transportation Research Record 2196(1): 65-74.

\section{About the Authors}

XIN ZHANG (leadaming@gmail.com) received an M.Sc. in Geographic Information Science from Peking University, China, and a Ph.D. in Civil Engineering from the University of Maryland, with a dissertation on developing an integrated model for coordinating mixed pedestrian-vehicle flows in metropolitan areas under emergency evacuation situations. His research interests include operational research modeling application in transportation, traffic simulation modeling, machine learning analysis on traffic data, and evacuation planning modeling. His current work is focused on operational research modeling on logistics and transit systems such as railways and public transportation.

GANG-Len ChANG is a professor in the Department of Civil and Environmental Engineering at the University of Maryland, College Park. He received an M.S. degree from National Chiao Tung University, Taiwan, and a Ph.D. from the University of Texas at Austin. His research interests include network traffic control, freeway traffic management and operations, realtime traffic simulation, and dynamic urban systems. His ongoing research projects include multimodal evacuation modeling for Baltimore City, applied technology and traffic analysis program (ATTAP), and traffic monitoring system for the Ocean City region. 


\section{SUBSCRIPTIONS and PREVIOUS ISSUES}

To subscribe or update your subscription to the Journal of Public Transportation, please visit www.nctr.usf.edu/journal/subscriptions.

Full-text issues of previous Journal of Public Transportation articles are available on the National Center for Transit Research website at www.nctr.usf.edu/journal/full-text-issues.

\section{SUBMISSION OF MANUSCRIPTS}

The Journal of Public Transportation is a quarterly, international journal containing original research and case studies associated with various forms of public transportation and related transportation and policy issues. Topics are approached from a variety of academic disciplines, including economics, engineering, planning, and others, and include policy, methodological, technological, and financial aspects. Emphasis is placed on the identification of innovative solutions to transportation problems.

All articles should be approximately 4,000 words in length (18-20 double-spaced pages). Manuscripts not submitted according to the journal's style will be returned. Submission of the manuscript implies commitment to publish in the journal. Papers previously published or under review by other journals are unacceptable. All articles are subject to peer review. Factors considered in review include validity and significance of information, substantive contribution to the field of public transportation, and clarity and quality of presentation. Copyright is retained by the publisher, and, upon acceptance, contributions will be subject to editorial amendment. Authors will be provided with proofs for approval prior to publication.

\section{Formatting Requirements}

All manuscripts must be submitted electronically in MS Word format, with no linked images. Tables in the manuscript must be created in Word or Excel format and must not be inserted as images. Figures should be inserted as images and, to meet Section 508 compliance requirements, must have alternative text (a thorough description of the image to assist persons with visual impairments in understanding the image). All tables and figures must be numbered consecutively and have a title. All non-original figures and graphics must indicate the source; the author should retain written permission for use of any non-original images.

All manuscripts should include sections in the following order, as specified:

- Cover Page - title (12 words or less), author(s) affiliation(s) and email address(es), and address/telephone for corresponding author

- Main Body - title, paper abstract (up to 150 words), text organized under section headings (do not use section or subsection numbering)

- References - author-date format (Chicago Manual of Style)

- Biographical Sketch - brief information for each author; include email address

Be sure to include the author's complete contact information, including email address, mailing address, telephone, and fax number.

\section{Submit manuscripts via email to:}

Lisa Ravenscroft, Assistant to the Editor

Journal of Public Transportation

National Center for Transit Research @ CUTR

Email: jpt@cutr.usf.edu

Phone: (813) 974-3120 